\title{
A Novel Saliva Collection Method among Children and Infants: A Comparison Study between Oral Swab and Pacifier-based Saliva Collection
}

\author{
Daniel Novak
}

\begin{abstract}
Aim: This study aims to test the feasibility and effectiveness of a novel pacifier-based saliva collection method on children and infants in comparison to an oral swab-based saliva collection method.

Materials and methods: This study was performed during spring 2018 in a clinical non-sponsored setting at Queen Silvia Children's Hospital pediatric emergency ward. Saliva collection was performed by comparing oral swab (Salimetrics ${ }^{\circledR}$ SalivaBio's Children's Swab) with a pacifier-based saliva collection method (Salivac ${ }^{\circledR}$ ). All participating children used both saliva collection systems. The amount of saliva collected in 2 minutes was measured. The amount of time needed for the healthcare professional was recorded. Parental preference was evaluated by a questionnaire. Results: No statistically significant difference was observed in collected saliva ( $174 \mu \mathrm{L}$ for pacifier-based saliva collection and $158 \mu \mathrm{L}$ for oral swab). The healthcare professional spent significantly less $(p<0.001)$ mean time with the pacifier-based saliva collection method than with the oral swab ( 31 vs $150 \mathrm{sec}$ ). A total of 48 out of the 52 caretakers preferred the pacifier-based saliva collection method compared to the oral swab. Conclusion: The novel pacifier-based saliva collection method proved to be a feasible, appreciated, and effective way of collecting saliva that simplifies the saliva collection method among children and infants.

Clinical significance: The pacifier-based saliva collection method simplifies saliva testing. The closed vacutainer system minimizes the risk of saliva contamination and opens up for novel home testing strategies.

Keywords: Child, Collection, Oral swab, Pacifier, Saliva.

The Journal of Contemporary Dental Practice (2021): 10.5005/jp-journals-10024-3028
\end{abstract}

\section{INTRODUCTION}

Invasive diagnostics tests, such as blood tests, are notoriously difficult to perform on children in both medical and dental practices. Non-invasive tests are preferred by the child, their caregivers, and the healthcare professional with an aim to minimizing the discomfort and simplifying the testing procedure.

Saliva testing is a non-invasive testing procedure that has received increased attention during the last years and with improved laboratory testing techniques, more analysis of saliva are being possible. ${ }^{1,2}$ Saliva has been suggested to play a vital role in future diagnostics of oral and systemic diseases. ${ }^{1}$ Saliva also plays an important role in the maintenance of oral and dental health and studies suggest numerous clinical situations that warrant saliva testing. ${ }^{3,4}$

Saliva collection among young children and infants is difficult since they have not yet learned to spit and the process of collecting saliva by passive drooling is too difficult. The current saliva collection technique among children and infants include cotton rope, syringe aspiration, filter paper, hydrocellulose microsponge, and oral swabs. ${ }^{5}$ All procedures require that the collection device be kept in the child's mouth for 1-3 minutes and that post-collection the saliva needs to be extracted from the collection device before analysis. All current methods are time-consuming with a need for concurrent supervision to minimize the risk of the child or infant choking on the collection device. The extraction of saliva from the collection device is also time-consuming and increases the risk of contamination of both the saliva and the healthcare professional. Another problem is to achieve
Department of Pediatrics, Institute for Clinical Sciences, Sahlgrenska Academy, University of Gothenburg and Queen Silvia Children's Hospital, Sahlgrenska University Hospital, Gothenburg, Sweden

Corresponding Author: Daniel Novak, Department of Pediatrics, Institute for Clinical Sciences, Sahlgrenska Academy, University of Gothenburg and Queen Silvia Children's Hospital, Sahlgrenska University Hospital, Gothenburg, Sweden, Phone: +46 313438072, e-mail:ddpnovak@gmail.com

How to cite this article: Novak D. A Novel Saliva Collection Method among Children and Infants: A Comparison Study between Oral Swab and Pacifier-based Saliva Collection. J Contemp Dent Pract 2021;22(1):9-12.

Source of support: Nil

Conflict of interest: None

a sufficient amount of saliva needed for the analysis within the time span the child or infant allows the collection device to be in their mouth. The goal is, therefore, to find a safe and time-efficient saliva collection technique that minimizes contamination, collects enough saliva, and above all is acceptable to both infants and children.

This study aimed to compare an oral swab saliva collection device with a novel pacifier-based saliva collection device among children and infants. In this comparison study, the two devices were compared in regards to the amount of saliva collected during the same time, the amount of work spent by a healthcare professional, and also the acceptance of the device.

(0) Jaypee Brothers Medical Publishers. 2021 Open Access This article is distributed under the terms of the Creative Commons Attribution 4.0 International License (https://creativecommons.org/licenses/by-nc/4.0/), which permits unrestricted use, distribution, and non-commercial reproduction in any medium, provided you give appropriate credit to the original author(s) and the source, provide a link to the Creative Commons license, and indicate if changes were made. The Creative Commons Public Domain Dedication waiver (http://creativecommons.org/publicdomain/zero/1.0/) applies to the data made available in this article, unless otherwise stated. 


\section{Method}

\section{Participants}

Children and infants were recruited at the emergency ward at Queen Silvia Children's University Hospital, Gothenburg, Sweden between February and April 2018. Inclusion criteria were children and infants under six years of age visiting the emergency ward with both caregivers. Exclusion criteria were children with an oral-facial malformation that did not allow pacifier use or children with salivary gland disorders, or previously collected saliva by one of the two devices. Both caregivers were informed about the study, orally and by written text. Infants were defined as children under the age of 12 months. A total of 20 infants and 30 children under the age of six years were recruited.

\section{Saliva Collection}

For the oral swab, the Salimetrics ${ }^{\circledR}$ SalivaBio's Children's Swab (SCS) was used. In accordance with the manufacturer's instructions, the oral swab was placed in the child's mouth for exactly 2 minutes. The author had to hold the swab in his hand the whole time to confirm the swab stayed in the child's mouth, collecting saliva and preventing it from being swallowed. After the saliva collection was done the swab was inserted into a $5 \mathrm{~mL}$ syringe and compressed to expel the total amount of saliva collected. The saliva was then measured in $\mu \mathrm{L}$ by a pipette.

The pacifier-based collection device $\left(\right.$ Salivac $\left.^{\circledR}\right)$ is designed as an ordinary pacifier but enables saliva to be collected within the device by the child or infant sucking on the pacifier. The pacifier was placed in the child's mouth for exactly two minutes. The pacifier-based collection device was then connected to a vacutainer system enabling the collected saliva to be transferred to a standard vacutainer by means of pressure difference. The saliva is therefore transported within a closed system minimizing contamination. Collected saliva in the vacutainer was extracted by means of a pipette and the amount of saliva measured in $\mu \mathrm{L}$.

Both saliva collection methods were used on each participating child. After one device was used, the child was allowed to rest for 5 minutes to allow the mouth to achieve normal saliva status again. After the rest, the other device was used. Since both devices were used in a consecutive manner on each child the order of which device that was used first was interchanged throughout the study. The reason for this was to prevent the possibility of the second device collecting less saliva because of a tired and less cooperative child. Half the time the swab was used first and half the time the pacifier was used first.

The time consumed for the healthcare professional for each device was measured in minutes and it was the actual time the healthcare professional needed to hold the device.

\section{Questionnaire}

Both caregivers were asked to answer a short questionnaire, consisting of 4 questions after both collection procedures were performed. The questionnaire contained questions regarding the child's prior experience of the pacifier and how long the child had been breastfeeding. The questionnaire also contained questions regarding how the caregivers perceived the collection devices to be and which saliva collection device the caregivers believed their child liked the most.

\section{Statistical Methods}

We tested for significant differences in mean saliva values and mean time consumed using t-tests and $\chi 2$ tests for categorical outcomes. All $p$-values are two-sided and values of $<0.05$ were considered statistically significant.

\section{Ethical Considerations}

The study was approved by the regional ethical review board and performed in accordance with the Declaration of Helsinki. It was emphasized that participation in the study was voluntary and that they could discontinue the study at any stage and their current treatment at the emergency ward was not affected by their participation or not participating in the study.

\section{Results}

A total of 52 children (aged 2-30 months) participated in the study which included collecting saliva by both methods and also answering the questionnaire. No one declined for the study. A total of 22 infants and 30 children under the age of six years participated. The study group consisted of 24 girls and 28 boys. A total of 31 of 52 children were previously or currently pacifier users. The results from both saliva collection methods are shown in Table 1. A mean of $158 \mu \mathrm{L}$ was collected by the oral swab and a mean of $174 \mu \mathrm{l}$ was collected with the pacifier-based collection device. Figure 1 shows the difference in the mean amount of saliva collected with each method. There was no significant difference in the amount of saliva

Table 1: Results for 52 children testing both saliva collection methods. Collection time 2 minutes

\begin{tabular}{lccc}
\hline & $\begin{array}{l}\text { Salimetrics }{ }^{\circledR} \text { SalivaBio's } \\
\text { Children's Swab }\end{array}$ & Salivac $^{\oplus}$ & \\
\hline $\begin{array}{l}\text { Average age } \\
\text { (months) }\end{array}$ & 23 & 23 & n.s. \\
$\begin{array}{l}\text { Previous pacifier } \\
\text { experience (\%) }\end{array}$ & 60 & 60 & n.s. \\
$\begin{array}{l}\text { Mean saliva } \\
\text { collected ( } \mu \text { L) }\end{array}$ & $158(0-350)$ & $174(0-300)$ & n.s. \\
$\begin{array}{l}\text { Mean time } \\
\text { needed for } \\
\text { healthcare } \\
\text { professional (sec) }\end{array}$ & 150 & 31 & $p<0.001$ \\
$\begin{array}{l}\text { Preference of } \\
\text { saliva collection } \\
\text { method (\%) }\end{array}$ & 8 & & \\
\hline
\end{tabular}

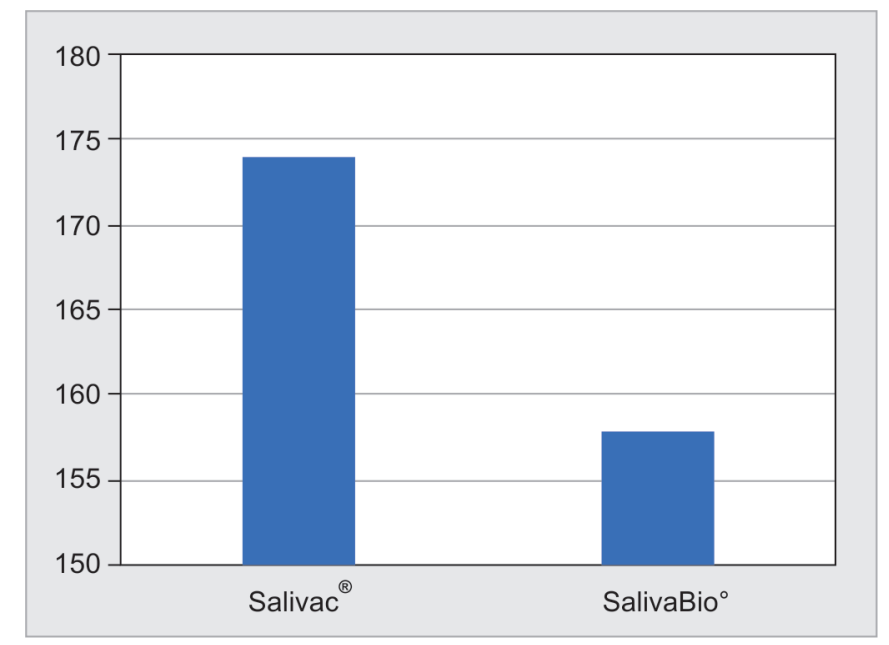

Fig. 1: Amount of saliva collected after 2 minutes use in $\mu \mathrm{L} . N=52$. Difference non-significant 
collected between the two methods when collecting for 2 minutes. The range was from $0 \mu \mathrm{L}$ in both devices to a maximum of $350 \mu \mathrm{L}$ in the pacifier-based collection device compared to the $300 \mu \mathrm{L}$ in the oral swab. One child did not want to take the pacifier. In four cases we could not retrieve any saliva with neither of the methods. All these unsuccessful cases were among infants aged five months or less. The final sample size of children collecting saliva was therefore 47 children (17 infants and 30 children). A total of 48 out of the 52 caregivers preferred the pacifier-based saliva collection device over the oral swab ( $p$-value $<0.001$ ).

In a subsample of 15 children, the pacifier was used a second time for four minutes, from which the amount of saliva was collected. The reason for this was to explore if the amount of saliva could increase with an increased amount of collection time. In all but two of the children, the saliva amount was increased by at least $100 \mu \mathrm{L}$. In one of the children, the whole pacifier-based saliva collection procedure was performed while the child was sleeping.

The time consumed for healthcare professional performing the saliva collection process varied between methods (Table 1). A significantly less amount of time $(p<0.001)$ was spent while using the pacifier-based saliva collection method (31 vs 150 mean seconds). When using the oral swab the healthcare professional continuously needed to hold the oral swab in his/her hand to stimulate the child to suck on the swab but also to prevent the child from swallowing it. The time needed to centrifuge the saliva to extract the saliva from the swab was not measured.

The author hypothesized that the pacifier-based saliva collection method was more user-friendly for both the healthcare professional and the child compared to the oral swab.

\section{Discussion}

Saliva is gaining increasing attention as a non-invasive sample medium in endocrinology, virology, pediatrics, and dentistry. ${ }^{6}$ The golden standard of saliva collection is passive drool but this is however not possible among children and infants. ${ }^{6}$ It is therefore vital that the healthcare system finds alternative saliva collection methods. This study compared two saliva collection techniques in infants and children in a clinical setting, oral swab vs pacifier-based saliva collection. The Salimetrics ${ }^{\circledR}$ SCS has been used successfully during recent studies collecting saliva in various community settings among children younger than five years. ${ }^{7,8}$ The saliva collection time for SCS is 2 minutes which according to Tryphonopoulos et al. may be too long for infants to tolerate, which may decrease their compliance. ${ }^{5}$ To my knowledge there has not been any previous published scientific article describing saliva collection by means of a pacifier nor its comparison to swab-based saliva collection.

The study showed that there was no significant difference in the amount of saliva collected between the two methods but significantly less time was needed for the healthcare professional when using the pacifier-based saliva collection method. The time consumption for the healthcare professional was a mean of 30 compared to 150 seconds with the oral swab $(p<0.001)$. This extended time consumption needed for the oral swab was twofold. Firstly, because the healthcare professional needed to hold the swab during the whole collection process to confirm the presence of the swab in the child's mouth but also to prevent the child from choking on the swab. Secondly, with the oral swab, the saliva needs to be extracted before analysis. In this study, the saliva was extracted by a syringe but if one should extract saliva the correct way the saliva would have to be centrifuged for 15 minutes at $1500 \times \mathrm{g}$ in accordance with the product manual. This can only be done by an educated healthcare professional with access to a laboratory and would require substantially more time. This saliva extraction step is not required when using a pacifier-based saliva collection method. The saliva is quickly and safely transferred to a vacutainer. This transfer of saliva does not need any healthcare education and can be done by a one-hand grip by a caretaker. This simplifies the saliva collection procedure. In future saliva collection scenarios, Salivac ${ }^{\circledR}$ can be used independently by the caregivers in the privacy of their own homes without the risk of contaminating the saliva sample.

The closed vacutainer system minimizes the risk of contaminating the saliva or the person collecting the saliva. The importance of not contaminating the saliva has increased with improved laboratory analysis especially saliva DNA. ${ }^{9}$ With the ability to connect the pacifier-based saliva collection device to a vacutainer prevents any contamination of the saliva or the testing person (caregiver or healthcare professional). The vacutainer can also be prefilled with a transport medium, such as nuclear acid stabilization media that has demonstrated cytomegalovirus (CMV) stability for up to three weeks. ${ }^{10}$ Transport or stability medium is often substances that could be irritating to both skin and mucous membranes and should only be used in laboratories with ventilation facilities. However, a prefilled vacutainer with a transport or stability medium would not allow the child or the person performing the saliva collection to come in contact with the substances. The advantage of having a prefilled vacutainers is that it enables saliva to be posted to a laboratory that facilitates the possibility of saliva collection at home. The importance of using a saliva collection device that does not risk contaminating the person handling the saliva cannot be emphasized enough during the 2019-nCoV epidemic. ${ }^{11}$

Both saliva collection methods were new and unfamiliar with the children participating in the study. The caregivers needed to engage themselves more in the oral swab collection to prevent the child from spitting it out. Many times saliva was collected from a crying mouth while using the oral swab. If this was due to the unfamiliar healthcare professional who needed to be close to the child the whole time holding the swab or because the swab tasted or felt different is difficult to know. However, less engagement was needed from the caregivers during the pacifier-based saliva collection procedure. In the initial phase, the child was hesitant due to the new look of the pacifier but after an often brief inspection it was inserted in the mouth and saliva collection began. Sometimes the testing device was extracted by the child, observed again, and then re-entered into the mouth. All this while, the healthcare professional was sitting at a safe distance observing. At the beginning of the design process of the pacifier, before the study was performed, the author noticed that it was important for the device to be light. A top-heavy device would push the pacifier to the palate that would cause discomfort for the child and no natural sucking would be achieved.

Even though only $60 \%$ of the participating children (31/52) had any previous experience of pacifiers $92 \%$ of the caregivers preferred the pacifier-based saliva collection method compared to the oral swab $(p<0.001)$. Only one child refused the pacifier. The reason for the high acceptance rate might be that the format of the pacifier-based saliva collection device might have been familiar to the children, which is an important aspect in making the child comfortable and cooperative. Sucking on a pacifier is a non-nutritive behavior using inborn reflexes and is frequently used to calm and soothe infants. The prevalence of pacifier use varies between 
countries and cultures but a recent prospective study for Danish children showed that $58 \%$ of them used pacifiers regularly. ${ }^{12}$ There have also been extensive discussions regarding both advantages and disadvantages of long term use of pacifiers. ${ }^{13}$ There should, however, not be any health disadvantage of using this pacifier-based saliva collection device since it is only for short term use.

Both saliva collection devices used stimulated saliva. The golden standard is passive drool, which is not possible to achieve among children and infants. The accuracy of stimulated saliva, especially in hormone analysis, has been questioned in multiple studies. 2,14,15 There are, however, several saliva analyses where stimulated saliva is acceptable. Saliva collection for virus analysis in dental practice does not depend on passive or stimulated saliva. ${ }^{16}$ Oral saliva microbiomes have shown to be minimally affected by different collection methods. ${ }^{17}$ Saliva has also been suggested as rapid identification of streptococci or influenza virus ${ }^{18,19}$ and even proven to be comparable to nasopharyngeal swab specimens in multiple viruses. ${ }^{20}$ A pediatric saliva collection device, therefore, needs to be simple, with high participation compliance and also minimizes the contamination risk of sample and sample handler.

The limitation of the study was the small sample size and that the saliva was not analyzed. The purpose of the study was however to investigate the acceptance of the device and also compare its saliva collection effectiveness to swab collection. Future studies need to analyze the saliva for relevant hormones, viruses, or bacteria.

\section{Conclusion}

This study leads to new knowledge in saliva collection within the pediatric population. This novel pacifier-based saliva collection simplifies saliva collection among children and infants. It proved to be a more feasible, more effective, and more accepted way of collecting saliva than the current saliva collection methods. Compared to the oral swab, it collects the same amount of saliva but with a significantly less amount of time spent by the healthcare professional. The closed vacutainer system minimizes the risk of contaminating the saliva or the person collecting the saliva and opens up for novel home testing strategies.

\section{Acknowledgment}

I wish to thank Prof Roger Karlsson the co-inventor of the pacifierbased collection device for helpful ideas during the design of the pacifier and study.

\section{References}

1. Khan RS, Khurshid Z, Yahya Ibrahim Asiri F. Advancing point-ofcare $(\mathrm{PoC})$ testing using human saliva as liquid biopsy. Diagnostics 2017;7(3):39. DOI: 10.3390/diagnostics7030039. PubMed PMID: 28677648; PubMed Central PMCID: PMCPMC5617939.

2. Buttler RM, Bagci E, Brand HS, et al. Testosterone, androstenedione, cortisol and cortisone levels in human unstimulated, stimulated and parotid saliva. Steroids 2018;138:26-34. DOI: 10.1016/j. steroids.2018.05.013. PubMed PMID: 29864449.

3. Ranganath LM, Shet RG, Rajesh AG. Saliva: a powerful diagnostic tool for minimal intervention dentistry. J Contemp Dent Pract 2012;13(2):240-5. DOI: 10.5005/jp-journals-10024-1130. PubMed PMID: 22665757.

4. Al Kawas S, Rahim ZH, Ferguson DB. Potential uses of human salivary protein and peptide analysis in the diagnosis of disease. Arch Oral Biol 2012;57(1):1-9. DOI: 10.1016/j.archoralbio.2011.06.013. PubMed PMID: 21774913.

5. Tryphonopoulos PD, Letourneau N, Azar R. Approaches to salivary cortisol collection and analysis in infants. Biol Res Nurs
2014;16(4):398-408. DOI: 10.1177/1099800413507128. PubMed PMID: 24136995.

6. Groschl M. Saliva: a reliable sample matrix in bioanalytics. Bioanalysis 2017;9(8):655-68. DOI: 10.4155/bio-2017-0010. PubMed PMID: 28504570.

7. Bhopal S, Verma D, Roy R, et al. The contribution of childhood adversity to cortisol measures of early life stress amongst infants in rural India: findings from the early life stress sub-study of the SPRING cluster randomised controlled trial (SPRING-ELS). Psychoneuroendocrinology 2019;107:241-250. DOI: 10.1016/j. psyneuen.2019.05.012. PubMed PMID: 31174162; PubMed Central PMCID: PMCPMC6642338.

8. Pisanic N, Ballard SB, Colquechagua FD, et al. Minimally invasive saliva testing to monitor norovirus infection in community settings. J Infect Dis 2019;219(8):1234-1242. DOI: 10.1093/infdis/jiy638. PubMed PMID: 30517651; PubMed Central PMCID: PMCPMC6452293.

9. Nunes LA, Mussavira S, Bindhu OS. Clinical and diagnostic utility of saliva as a non-invasive diagnostic fluid: a systematic review. Biochem Med 2015;25(2):177-192. DOI: 10.11613/BM.2015.018. PubMed PMID: 26110030; PubMed Central PMCID: PMCPMC4470107.

10. Goshen O, Goldfarb DM, Book L, et al. Recovery of cytomegalovirus DNA from newborn saliva samples by different methods. J Clin Virol 2018;104:73-76. DOI: 10.1016/j.jcv.2018.05.001. PubMed PMID: 29753934.

11. Xu R, Cui B, Duan X, et al. Saliva: potential diagnostic value and transmission of 2019-nCoV. Int J Oral Sci 2020;12(1):11. DOI: 10.1038/ s41368-020-0080-z. PubMed PMID: 32300101; PubMed Central PMCID: PMCPMC7162686.

12. Maastrup $\mathrm{R}$, Hansen BM, Kronborg $\mathrm{H}$, et al. Factors associated with exclusive breastfeeding of preterm infants. Results from a prospective national cohort study. PLoS One 2014;9(2):e89077. DOI: 10.1371/journal.pone.0089077. PubMed PMID: 24586513; PubMed Central PMCID: PMCPMC3929624.

13. Schmid KM, Kugler R, Nalabothu P, et al. The effect of pacifier sucking on orofacial structures: a systematic literature review. Prog Orthod 2018;19(1):8. DOI: 10.1186/s40510-018-0206-4. PubMed PMID: 29532184; PubMed Central PMCID: PMCPMC5847634.

14. Groschl M, Rauh M. Influence of commercial collection devices for saliva on the reliability of salivary steroids analysis. Steroids 2006;71(13-14):1097-1100. DOI: 10.1016/j.steroids.2006.09.007. PubMed PMID: 17070563.

15. Shirtcliff EA, Granger DA, Schwartz E, et al. Use of salivary biomarkers in biobehavioral research: cotton-based sample collection methods can interfere with salivary immunoassay results. Psychoneuroendocrinology 2001;26(2):165-173. DOI: 10.1016/s03064530(00)00042-1. PubMed PMID: 11087962.

16. Corstjens PL, Abrams WR, Malamud D. Detecting viruses by using salivary diagnostics. J Am Dent Assoc 2012;143(10 Suppl):12S-18S. DOI: 10.14219/jada.archive.2012.0338. PubMed PMID: 23034833; PubMed Central PMCID: PMCPMC4262792.

17. Lim $Y$, Totsika $M$, Morrison $M$, et al. The saliva microbiome profiles are minimally affected by collection method or DNA extraction protocols. Sci Rep 2017;7(1):8523. DOI: 10.1038/s41598-017-07885-3. PubMed PMID: 28819242; PubMed Central PMCID: PMCPMC5561025.

18. Lee JW, Jung JY, Lim SK. Simple and rapid identification of saliva by detection of oral streptococci using direct polymerase chain reaction combined with an immunochromatographic strip. Forensic Sci Int Genet 2018;33:155-160. DOI: 10.1016/j.fsigen.2017.12.011. PubMed PMID: 29289820.

19. Yoon J, Yun SG, Nam J, et al. The use of saliva specimens for detection of influenza $A$ and $B$ viruses by rapid influenza diagnostic tests. J Virol Methods 2017;243:15-19. DOI: 10.1016/j.jviromet.2017.01.013. PubMed PMID: 28111058.

20. Kim YG, Yun SG, Kim MY, et al. Comparison between saliva and nasopharyngeal swab specimens for detection of respiratory viruses by multiplex reverse transcription-PCR. J Clin Microbiol 2017;55(1):226-233. DOI: 10.1128/JCM.01704-16. PubMed PMID: 27807150; PubMed Central PMCID: PMCPMC5228234. 